Running title: SHORT-TERM MEMORY FOR FACES IN DYSPHORIA

\title{
Short-term memory for emotional faces in dysphoria
}

Word Count: 5331 (excluding the abstract, references and tables)

Saima Noreen and Nathan Ridout

Clinical \& Cognitive Neurosciences,

Aston University, Birmingham, UK

Address for correspondence:

Dr Nathan Ridout

Clinical \& Cognitive Neurosciences

School of Life \& Health Sciences

Aston University

Birmingham, B7 4ET

Tel: (+44 121) 2044162

Fax: (+44 121) 2044090

Email: n.ridout@aston.ac.uk 


\begin{abstract}
The aim was to determine if the memory bias for negative faces that has been demonstrated in depression and dysphoria generalises from long to short-term memory. 29 dysphoric (DP) and 22 non-dysphoric (ND) participants were presented with a series of 64 faces, each featuring one of four emotional expressions (happiness, sadness, anger or neutral affect), and were asked to identify the emotion portrayed. Following a short delay, an array of four faces (the original plus three distracters) was presented and the participants were asked to identify the target face. Half the arrays featured the same individual with four different expressions (facial expression memory) and half featured four different individuals with the same expression (facial identity memory). At encoding, no group differences were apparent. At memory testing, DP exhibited impaired memory for all types of facial emotion and for facial identity when the faces featured happiness, anger or neutral affect, but not when the faces featured sadness. DP exhibited enhanced memory for faces with sad \& angry expressions, and impaired memory for happy faces, relative to neutral. ND exhibited enhanced memory for facial identity when faces were angry, relative to sad or neutral. The implications of these findings are discussed in terms of the literature on MCM biases and social functioning in depression.
\end{abstract}

Key words: Dysphoria; Depression; Mood-Congruent Memory; Emotional Facial Expressions; Short-term Memory; Delayed-Matching-to-Sample. 


\section{INTRODUCTION}

Previous work has revealed that clinical depression is associated with a negative memory bias for emotional faces. For example, Ridout, Astell, Reid, Glen \& O'Carroll (2003) reported that depressed patients exhibited enhanced recognition memory for sad faces relative to happy or neutral. A similar memory bias for angry faces has also been reported in depressed patients (Gilboa-Schechtman et al.., 2002). More recently, an equivalent bias has been demonstrated in individuals experiencing naturally occurring sub-clinical depression (referred to as dysphoria; Jermann, van der Linden \& D’ Argembeau, 2008; Ridout, Noreen \& Johal, in press) and in participants in an induced depressed mood (Ridout et al., in press). These findings contribute to a wider literature demonstrating alterations to normal face processing in depressed and dysphoric individuals. For example, there is evidence that depression is associated with impaired facial emotion recognition (e.g. Leppänen, Milders, Bell, Terriere \& Hietanen, 2004; Persad \& Polivy, 1993; Surguladze et al., 2004). Further, there is growing evidence that depressed individuals selectively attend to negative faces, relative to positive or neutral (e.g. Bradley et al., 1997; Gotlib, Krasnoperova, Yue \& Joorman, 2004; Gotlib et al., 2004b).

It is well known that depression and dysphoria are associated with impaired social functioning (Gotlib \& Lee, 1989; Harkness, Sabbagh, Jacobson, Chowdrey \& Chen, 2005; Joiner \& Coyne, 1999; Petty, Sachs-Ericsson \& Joiner, 2004). It has been suggested that alterations in face processing may contribute to, or even account for, impaired social functioning in depression (Persad \& Polivy, 1993; Ridout et al., 2009). In line with this notion, the memory bias for sad and angry faces observed in depressed individuals could plausibly impact negatively on their social functioning. For example, such a memory bias could act colour the perception of interactions with 
significant others, leading to misunderstandings and potentially interpersonal conflicts, which in turn could weaken important social bonds. However, given that facial expressions change a number of times throughout the duration of a social interaction, a bias in short-term memory for emotional faces would arguably be of greater importance in terms of influencing social functioning. Therefore, the aim of the current study was to assess the effect of depression on short-term memory for emotional faces.

To date, the only study that has assessed short-term memory for emotional faces in depression was conducted by Deveney and Deldin (2004), who utilised a facial variant of the delayed-matching-to-sample task (DMTS). Although they reported that non-depressed participants exhibited reduced slow wave EEG activity for sad faces relative to happy and neutral, their results did not reveal group differences in memory for emotional faces. However, there were a number of limitations to their study that have to be addressed before it can be firmly concluded that depression does not influence short-term memory for emotional faces. As noted by the researchers themselves their working memory task may have been too easy to provide a sensitive measure of group differences in face memory. Consistent with this proposal it has been widely reported that depressed patients demonstrate greater impairment on tasks that make larger demands on cognitive resources than tasks that require little or no cognitive effort (Hartlage, Alloy, Vasquez \& Dykman, 1993; Williams, Watts, MacLeod, \& Mathews, 1997). A further issue concerns the encoding phase of Deveney and Deldin's study (2004). According to Teasdale \& Barnard's influential Interacting Cognitive Subsystems (ICS) model (1993) the presence of MCM biases is dependent upon the affective content of the stimuli being explicitly processed at encoding. Thus, as the participants in Deveney and Deldin's study were 
not required to overtly process the emotional content of the faces, a MCM bias would not have been expected. Consistent with this notion, Ridout et al.. (2009) reported that clinically depressed patients did not exhibit a bias for sad faces following a nonemotional encoding task (gender identification). On the other hand, all studies that have successfully demonstrated MCM biases for negative faces in depressed states have involved the participants overtly processing the emotional element of the faces at encoding (Gilboa-Schechtman et al.., 2002; Jermann et al., 2008; Ridout et al.., 2003; in press). Taken together these findings support the importance of explicit emotional processing at encoding in the emergence of MCM biases.

Another issue of importance concerns the distinction between memory for facial identity and memory for facial emotion. Models of face processing generally assume that identity recognition and expression recognition involve independent subprocesses which are neuropsychologically and experimentally distinct (Bruce \& Young, 1986; Haxby, Hoffman \& Gobbini, 2000). However, recent research suggests that there may be an interaction between the processing of emotional expression and facial identity, with memory for an individual's identity and recall of their emotional expression both being influenced by the nature of the facial expression (Cohen-Pager \& Brosgole, 1992; D’Argembeau, Van der Linden, Comblain \& Etienne, 2003; Foa, Gilboa-Schechtman, Amir \& Freshman, 2000). Further, Jermann et al.. (2008) reported that dysphoria influenced memory for emotional expressions, but not facial identity. However, their study utilised a long-term memory task, thus it is not known if this distinction would hold in dysphoric individuals' short-term memory.

The aim of the present study was to utilize a complex working memory task (that involves explicit processing of the emotional content of the faces at encoding) in order to determine if dysphoric individuals exhibit a short-term mood congruent 
memory (MCM) bias for negative emotional faces. A further aim was to establish whether the type of emotional expression biased participants' memory for the facial identity, the emotional expression, or both of these elements of the faces. Thus, groups of dysphoric and non-dysphoric participants were presented with a series of photographs of faces exhibiting one of four emotions (happiness, anger, sadness or neutral affect) and were asked to identify the emotion being portrayed. Following a short delay, participants were presented with an array of four faces and were required to identify the face they had seen at encoding. Half of these arrays featured the same individual with four different expressions (expression memory test) and half featured four different individuals with the same expression (identity memory test). It was predicted that, during the recognition memory element of the task, dysphoric individuals would remember the identity of significantly more of the faces that portrayed sadness than happiness or neutral affect. Furthermore, based on previous findings (Gilboa-Schechtman et al.., 2002) it was also expected that dysphoric individuals would remember the identity of significantly more of the faces that portrayed anger than happiness or a neutral expression. Conversely, it was predicted that individuals with low BDI scores would remember the identity of significantly more of the faces that expressed happiness than sadness, anger or neutral affect. In terms of memory for the emotional expression, it was predicted that dysphoric individuals would show enhanced memory for sadness and anger rather relative to happiness or neutral affect. Conversely, it was expected that participants with low BDI scores would show superior memory for happiness relative to sadness, anger or neutral affect. 


\section{METHOD}

\section{Participants}

29 dysphoric ( 9 males, 20 females; mean age $=23.3$ years, $\mathrm{SD}=2.9$ ) and 22 nondysphoric ( 8 males, 14 females; mean age $=22.5$ years, $\mathrm{SD}=3.0)$ participants took part in the current study. Participants were recruited through Aston University's research participation scheme and received course credit for their participation. They were allocated to one of the two groups based on their scores on the latest version of the Beck Depression Inventory (BDI-II; Beck, Brown and Steer, 1996). Following Walker, Skowronski, Gibbons, Vogl and Thompson (2003) participants with BDI scores of 0 - 9 were classified as non-dysphoric and participants with scores of 10+ were classified as dysphoric. All participants were medication-free, had normal or corrected-to-normal vision and were right handed. All participants provided full written informed consent prior to taking part in the study, but were not informed of the true nature of the study until the debriefing session. The study was approved by Aston University's research ethics committee.

\section{Self-reported Mood}

The latest version of the Beck Depression Inventory (BDI-II; Beck et al.., 1996) consists of 21-items which assess the presence and severity of the symptoms of depression and was used in the present study to classify participants into the dysphoric or non-dysphoric groups (see participants section for criterion). The Spielberger State-Trait Anxiety Inventory (STAI; Spielberger, 1983) was used in the present study to identify the presence and severity of current (state) and dispositional (trait) anxiety. The STAI was also used to control for the effects of anxiety on 
cognitive processing, as anxiety has also been shown to influence memory function (see Williams et al.., 1997 for a review).

\section{Neuropsychological Measures}

Prior investigations have found that both depression and dysphoria impair performance on various aspects of neuropsychological functioning, including psychomotor speed, attention and memory (Ilsley et al.., 1995; Channon et al.., 1999; Jogems-Kosterman et al.., 2001). Therefore, a battery of tests tapping a number of neuropsychological domains was included in the present study in order to preclude general deficits in cognitive performance as an explanation for any observed memory biases. Psychomotor retardation was assessed using the Digit Symbol Substitution Task (DSST) from the Wechsler adult intelligence scale (WAIS; Wechsler, 1981). Executive function was assessed using the FAS Verbal Fluency Task (Spreen \& Strauss, 1998) and working memory and visual-spatial attention were assessed using the Digit and Spatial Span Tasks from the WAIS (Wechsler, 1981).

\section{Facial Stimuli}

The facial stimuli presented during the emotion labeling (encoding) phase of the study were a series of 32 gray-scale images taken from Ekman \& Friesen's (1975) Pictures of Facial Affect (see Figure 1 for an example). These 32 images consisted of photographs of 8 individuals ( 4 female $\& 4$ male) portraying four different emotions (happiness, sadness, anger and neutral affect). Hair was digitally removed from the images to reduce the distinctiveness of faces. In addition, 8 images of two individuals (both female) portraying the four emotions were used for the practice phase of the 
task. The facial stimuli presented during the memory testing phase consisted of 64 arrays, each featuring four faces presented side by side horizontally across the screen (see Figure 1 for an example). The faces in each array were labeled A to D from left to right. In order to distinguish the effects of emotional expression on memory for facial identity and facial expression, half of the 64 arrays featured four different individuals portraying the same emotion. The remaining 32 displays consisted of the same individual portraying the four different emotions. In addition, a further 8 arrays were constructed for the practice phase.

\section{Neuropsychological Assessment}

Once informed consent had been obtained, the participants were asked to complete general neuropsychological assessment measures in the following sequence: Digit Symbol Substitution Task (DSST), FAS Verbal Fluency Task (VFT), Digit \& Spatial Span tasks (forwards \& backwards). All tasks were conducted according to their standard instructions.

\section{Emotional Identification and Facial Working Memory Tasks}

This main experimental session consisted of 64 trials (plus 16 practice trials). Each trial (see Figure 1) began with a black fixation cross (size 24 font Arial) being presented in the centre of the screen for $700 \mathrm{~ms}$. Participants were instructed to maintain their attention on the cross at the start of each trial. A single image of an individual depicting one of four emotions (happiness, sadness, anger, or neutral affect) was then displayed for 2500 milliseconds. The participant's task was to identify the emotion that the face was eliciting by pressing the labeled computer key 
that corresponded to the emotion. Following the offset of the image another visual display appeared in the form of a scrambled face (see Figure 1 for an example) which was presented for 15 seconds. Participants were required to maintain their attention upon this stimulus for the duration it remained on the screen. Following the delay period an array of four faces was presented, featuring the target face (viewed at encoding) and three distracters. Participants were asked to identify which of the four faces they had viewed just prior to the delay period by pressing the key labeled with the appropriate letter (e.g. A for position one in the array, B for the second position and so on). The array remained on the screen until the participant responded. However, retrieval time was controlled by asking participants to respond as quickly and accurately as possible. The position of the correct emotional face in the memory recognition phase was counterbalanced across trials so that each face appeared equally often in each of the four locations. SuperLab Pro (version 2.0.4; Cedrus Corporation, 1999) was used to control stimulus presentation and to record response accuracy and latency. The facial stimuli were presented on a colour monitor that was $18 \mathrm{~cm}$ (height) $* 25 \mathrm{~cm}$ (width) in size. The size of the stimuli when presented on the screen was $10 \mathrm{~cm}$ (width) $* 15 \mathrm{~cm}$ (height) and the participants were seated approximately $50 \mathrm{~cm}$ from the screen. All trials were presented in a new, fully randomized order for each participant.

\section{Scoring and Data Analysis}

Group differences in age and scores on the BDI (depression) and STAI (state and trait anxiety) were analysed using independent t-tests. Group performance on the neuropsychological test battery was assessed using a one-way between-groups multivariate analysis of variance. The ratio of males and females in each group was 
analysed using a 2 x 2 chi-square. Data for the encoding and recognition memory phases, which included accuracy rates and average reaction times, were analyzed using a 2 x 4 mixed factorial ANOVA. The between-subjects factor was participant group (dysphoric vs. non-dysphoric) and the within-subjects factor was type of emotional expression (happy vs. angry vs. neutral vs. sad). The dependent variables were: $i$ ) emotion identification time (milliseconds) $i$ ) percentage of emotional expressions correctly labeled $\mathrm{iii}$ ) percentage of facial identities correctly recognized during memory testing, $i v$ ) percentage of facial expressions correctly recognized during memory testing, and $v$ ) time taken to make recognition memory decisions (milliseconds). The recognition memory data for arrays featuring four different individuals with the same expression and those featuring the same individual with four different expressions were analyzed separately, in order to distinguish the effects of facial emotion on memory for facial identity and memory for the emotion itself. Follow up analyses were conducted using one-way repeated measures ANOVA and independent samples t-tests. Alpha levels for pairwise comparisons were adjusted, where appropriate, in accordance to the Bonferroni correction method (see Keppel \& Wickens, 2004).

\section{RESULTS}

\section{Group Characteristics}

Analysis of the participant' characteristics (presented in Table 1) revealed that the dysphoric (D) and non-dysphoric (ND) groups did not differ significantly in terms of their age or gender ratio; $\mathrm{t}(49)=1.0, \mathrm{p}>0.05$ and $\chi^{2}(1) 1.2, \mathrm{p}>.05$ respectively. Further, no significant group differences were observed for state or trait anxiety; $\mathrm{t}(49)=0.2, \mathrm{p}>0.05$ and $\mathrm{t}(49)=.8, \mathrm{p}>0.05$ respectively. However, as expected the 
dysphoric individuals scored significantly higher on the depression (BDI-II) measure $($ Mean $=17.8, S D=7.2)$ than did the non-dysphoric participants $(M=4.9, S D=4.1)$; $\mathrm{t}(49)=8.1, \mathrm{p}<.001$.

\section{General Neuropsychological Assessment}

Analysis of the participants' performance on the battery of neuropsychological tests (presented in Table 2) revealed that two groups did not differ significantly in their general cognitive function; $F(6,44)=0.7, p>.05$ (Pillai's Trace). Note: no individual test for the different neuropsychological measures achieved, or even approached, statistical significance, suggesting the lack of group difference was observed across domains of cognitive function.

\section{Emotion Identification Time}

Analysis of the participants' emotion identification times revealed no significant differences between the dysphoric \& non-dysphoric groups and no group x type of emotion interaction; $\mathrm{F}(1,49)=0.12, \mathrm{p}>.05$ and $\mathrm{F}(1,49)=0.26, \mathrm{p}>.05$ respectively. However, there was a significant main effect of type of emotion, $F(1,49)=9.1, \mathrm{p}<$ .001. Subsequent analysis (using least significant difference tests) revealed that, overall, participants identified happy facial expressions more rapidly (Mean=571.12 milliseconds, $\mathrm{SD}=168)$ than neutral $(\mathrm{M}=677.91 \mathrm{~ms}, \mathrm{SD}=216.42)$, sad $(\mathrm{M}=644.68 \mathrm{~ms}$, $\mathrm{SD}=207.64)$, or angry facial expressions $(\mathrm{M}=699.99 \mathrm{~ms}, \mathrm{SD}=189.60)$; all tests $\mathrm{p}$ $<.001$

\section{Emotion Identification Accuracy}

Analysis of the percentage of emotional expressions correctly labeled revealed no significant difference between dysphoric \& non-dysphoric groups and no group x type of emotion interaction; $F(1,49)=1.22, p>.05$ and $F(1,49)=0.73, p>.05$ 
respectively. However, there was a highly significant main effect of type of emotion; $\mathrm{F}(1,49)=19.6, \mathrm{p}<.001$. Subsequent analysis (using least significant difference tests) revealed that, overall, participants correctly identified significantly more happy expressions $(\mathrm{Mean}=96.2 \%, \mathrm{SD}=7.3)$ than neutral $(\mathrm{M}=81.5 \%, \mathrm{SD}=18.7)$, sad $(\mathrm{M}=84.4 \%, \mathrm{SD}=13.3)$, or angry $(\mathrm{M}=82.0 \%, \mathrm{SD}=13.1)$; all tests $\mathrm{p}<.001$.

\section{Recognition Memory Latencies}

Analysis of the influence of emotional expressions and facial identity on recognition memory response times (data not shown) revealed no significant differences; all tests $\mathrm{p}>.05$.

\section{Memory for Emotional Expression}

Analysis of the participants' memory for the different emotional expressions (see Figure 2) revealed that non-dysphoric participants remembered significantly more facial expressions (Mean=92.2\%, Standard Error=3.8) than did dysphoric participants $(\mathrm{Mean}=80.5 \%, \mathrm{SE}=3.3) ; \mathrm{F}(1,49)=5.41, \mathrm{p}<.05$. Results also revealed that the type of emotion influenced the participants' memory for the expressions; $F(1,49)=6.32$, $p$ $<.001$. Follow up analyses revealed that, overall, participants remembered significantly more angry (Mean $=88.7 \%, \mathrm{SE}=3.4)$, sad $(\mathrm{Mean}=90.0 \%, \mathrm{SE}=2.3)$ and happy (Mean=86.5\%, SE=3.2) than neutral expressions (Mean=77.0\%, SE=2.5); all tests $\mathrm{p}<.001$. However, recognition memory for the three different emotional expressions (happiness, sadness \& anger) did not differ significantly; all tests $\mathrm{p}>.05$. Analysis of the participants' memory for the facial expressions revealed no significant group $\mathrm{x}$ type of emotion interaction; $\mathrm{p}>.05$. 
Analysis of memory for facial identity (see figure 3) revealed that non-dysphoric participants remembered more faces $($ Mean $=92.5 \%$, Standard Error $=4.6)$ than did dysphoric participants (Mean=77.4\%, $\mathrm{SE}=4.0) ; \mathrm{F}(1,49)=6.21 \mathrm{p}<.05$. Results also revealed a significant effect of type of emotion; $F(1,49)=3.41 \mathrm{p}<.05$. However, this finding should be considered in light of the significant group $\mathrm{x}$ type of emotion interaction; $\mathrm{F}(1,49)=3.41 \mathrm{p}<.05$. Within group comparisons revealed that the dysphoric participants remembered significantly more sad $(\mathrm{M}=80.2 \%, \mathrm{SE}=5.4)$ than happy faces $(\mathrm{M}=72.0 \%, \mathrm{SE}=5.4) ; \mathrm{p}<.01$. They also remembered significantly more angry $(M=79.3 \%, S E=3.0)$ than happy faces; $p<.05$. Further, dysphoric participants remembered more neutral faces $(\mathrm{M}=78.0 \%, \mathrm{SE}=5.5)$ than happy, a difference that approached conventional significance, $p=.08$. However, although the dysphoric participants exhibited better memory for sad and angry faces relative to neutral, this difference was non-significant, $\mathrm{p}>.05$. Analysis of the non-dysphoric participants' data revealed that they remembered significantly more angry $(M=96.6 \%, S E=2.0)$ than $\operatorname{sad}(\mathrm{M}=88.6 \%, \mathrm{SE}=3.3)$ or neutral faces $(\mathrm{M}=92, \mathrm{SE}=2.4) ; \mathrm{p}<.001$ and $\mathrm{p}<.05$ respectively. However, no other within group differences were apparent, all other tests p>.05. Between group comparisons revealed that when the faces featured happy, angry or neutral expressions the non-dysphoric participants remembered significantly more facial identities than did the dysphoric participants; $\mathrm{t}(49)=2.74, \mathrm{p}<.01, \mathrm{t}(49)=$ $2.88, \mathrm{p}<.01$ and $\mathrm{t}(49)=2.20, \mathrm{p}<.05$ respectively. However, when the faces were sad the two groups did not differ in terms of their memory for facial identity; $t(49)=1.49$, $\mathrm{p}>.05$. 


\section{DISCUSSION}

The primary aim of the present study was to determine if the negative memory bias that has been demonstrated in depressed and dysphoric individuals on explicit longterm memory tasks generalises to short-term memory. A further aim was to establish whether the type of emotional expression biased participants' memory for the facial identity, the emotional expression, or both of these elements of the faces. To these ends, dysphoric and non-dysphoric participants were assessed on a facial variant of the delayed matching-to-sample task.

Before considering the memory data it is necessary to consider possible group variations in processing at encoding. The present findings demonstrate that dysphoric individuals did not differ from non-dysphoric controls in their ability to identify emotion from facial expressions. This finding is inconsistent with many previous studies (e.g. Leppänen et al., 2004; Persad \& Polivy, 1993) but in line with other studies assessing emotion recognition ability in dysphoric (Frewen \& Dozois, 2005) and clinically depressed participants (Archer et al.., 1992; Ridout et al.., 2003). The current findings are not surprising given that recent research has implicated timerelated parameters in emotion recognition deficits in dysphoria and depression (Sato et al.., 2001). For example, a recent study by Surguladze et al.. (2004) found no difference between depressed individuals and non-depressed controls in emotion identification from facial expressions presented for $2000 \mathrm{msec}$, but significant impairments in depressed individuals' ability to identify sad and happy expressions when the faces were presented at short durations $(100 \mathrm{msec})$. Given that the facial expressions presented during the encoding phase of the present study were displayed for 2500 milliseconds, the present findings are wholly consistent with this pattern. 
The present findings also revealed a general (across groups) identification advantage for happy facial expressions (indicated by more rapid and accurate identification, relative to anger, sadness or neutral affect). This happy face advantage is a relatively robust phenomenon (Billings et al.., 1993; Crews \& Harrison, 1994; Hugdahl et al.., 1993; Ridout et al.., 2003), which may relate to differences in the visual features of positive and negative emotions. Negative emotions share changes in the configuration of facial features (Johnston et al.., 2001) and are processed in more complex ways than positive emotions (Baumeister et al.., 2001). It has been suggested that there is a greater tendency to utilise full visual analysis when processing negative faces, which places greater demands on the perceptual systems (Fabre-Thorpe et al.., 2001). With this in mind, it is plausible that the relative deficits in negative emotion recognition (slower identification times and lower accuracy rates) observed in the present study might be a consequence of this tendency.

At memory testing, it was predicted that dysphoric individuals would show enhanced memory for sad and angry expressions relative to happy or neutral. Conversely, it was expected that participants with low BDI scores would show superior memory for happy expressions relative to the other three expressions (sadness, anger \& neutral affect). Neither of these hypotheses were supported by the current data. However, the type of emotional expression did influence the participants' memory, as they correctly remembered more emotional than neutral facial expressions. This finding is consistent with a body of work reporting superior memory for emotional vs. neutral words, pictures, sentences, and narrated slide shows (Buchanan \& Adolphs, 2002; Hamann, 2001; Kensinger \& Corkin, 2003). The lack of MCM bias for negative expressions in the dysphoric participants is inconsistent with the findings of Jermann et al. (2008), as they reported a clear memory advantage 
for sad expressions relative to happy in their dysphoric sample. This suggests that the MCM bias does not generalize from long to short-term memory. However, the finding that dysphoric participants exhibited impaired short-term memory for all facial expressions relative to the non-dysphoric individuals is an interesting and important result. This memory deficit for recently experienced emotional expressions could have significant negative implications for these individuals' social functioning. For example, by leading to social misunderstandings and interpersonal conflicts that could weaken important social links. This is important, as previous work has identified poor social support as a significant factor in ongoing psychological distress and the onset of mental illness (Hipkins, Whitworth, Tarrier \& Jayson, 2004; Klineberg et al.., 2006; Lee et al.., 2006; Vaananen, Vahtera, Pentti \& Kivimaki, 2005). Thus, this memory deficit could represent a risk factor for the development of more serious depressive episodes.

It was also predicted that, at memory testing, dysphoric individuals would exhibit significantly better memory for the individuals expressing negative emotions (sadness \& anger) than happy or neutral expressions. Results provided partial support for this hypothesis, as dysphoric participants demonstrated superior memory for sad and angry faces relative to happy. These findings are largely consistent with studies that have assessed clinically depressed patients on long term memory tasks (GilboaSchechtman et al.., 2002; Ridout et al.., 2003) and suggest that the MCM bias generalizes from long to short-term memory. However, although the means were in the predicted direction, dysphoric participants did not show enhanced memory for negative faces relative to neutral, which is inconsistent with the previous work using LTM tests in depressed patients. However, this could relate to the tendency (observed in all participants) to label neutral faces as sad. Given that sad faces were remembered 
more readily by the dysphoric group, they would presumably have demonstrated a similar memory advantage for any neutral faces that were perceived as sad, which would have distorted the rate of recognition memory for neutral faces and masked the expected difference. The findings that dysphoric participants demonstrated enhanced memory for faces with angry and sad expression relative to happy is inconsistent with the findings of Jermann et al. (2008), as they reported that memory for facial identity was not influenced by emotional expression. Interestingly, there was some evidence from the current study that dysphoric participants remembered more faces with a neutral expression than happy. Taken together these results actually suggest that dysphoria is associated with impaired short-term memory for faces with happy expressions relative to faces with the other three expressions. This is also an important finding; as such a bias could potentially negatively influence these individuals' social functioning.

The prediction that non-dysphoric individuals would demonstrate enhanced memory for faces with a happy expression was not supported by the results of the current study. Interestingly, non-dysphoric participants demonstrated superior memory for faces with an angry expression relative to a sad or neutral expression. Recognition memory for angry and happy faces did not differ in the non-dysphoric participants. These findings are inconsistent with previous studies, which have reported enhanced memory for happy faces relative to angry (D'Argembeau et al.., 2003; Foa, Gilboa-Schechtman, Amir \& Freshman, 2000; Kottoor, 1989). A possible explanation for these findings could relate to the social/emotional meaning provided by emotional expressions. Facial expressions are powerful sources of information which enable individuals to infer feelings and intention of others towards themselves. Therefore it is possible that the cost of failing to remember the identity of someone 
with an angry or threatening facial expression is potentially higher than the cost associated with failing to remember the identity of some with a neutral or sad expression and thus attracts more attention leading to enhanced memory of angry faces (Eastwood, Smilek \& Merikle, 2003; Fox et al.., 2000; Ohman, Lundqvist, Esteves, 2001).

The finding that dysphoric participants demonstrated impaired memory for the identity of previously presented faces is notable, as deficits in the ability to recognise facial identity have been implicated in the development of social dysfunction (Phillips, 2004), which, as noted above, has negative implications for the development of psychological distress (Hipkins et al.., 2004; Klineberg et al.., 2006; Lee et al.., 2006; Vaananen et al.., 2005). Taken together these results suggest a specific deficit in face processing in the dysphoric group, which is consistent with findings in clinically depressed patients (Brown, Scott, Bench \& Dolan, 1994; Palmer et al.., 1996; Ridout et al.., 2009). However, it is notable that, although the dysphoric participants exhibited impaired memory for facial identity when the faces featured happy, neutral or angry expressions, the two group's memory performance did not differ when the faces featured a sad expression. This could plausibly relate to enhanced processing of sad faces on the part of the dysphoric participants due to their emotional salience. However, it could be an artifact due to slightly elevated memory for faces with a sad expression in the dysphoric group and marginally poorer memory for these faces in the non-dysphoric group.

The observed memory deficits cannot be accounted for by a general cognitive impairment in the dysphoric group, as the two groups did not differ in their performance on the battery of neuropsychological tests, including measures of visual and verbal short-term memory. However, it is plausible that other individual 
differences may have contributed to the findings. For example, there is considerable evidence that depressed mood is associated with a greater tendency to ruminate (e.g. Nolen-Hoeksema, 1991; Nolen-Hoeksema, Parker \& Larson, 1994). Rumination, defined as the focusing of one's thoughts on one's current mood, the causes of one's depressive symptoms and worrying about the implications of one's depressive symptoms (Watkins \& Brown, 2002), has been shown to impair working memory function (e.g. Joorman \& Gotlib, 2008). With this in mind, it is possible that during the 15 second delay between encoding a memory testing, the working memory of the dysphoric group may have been disrupted by processing ruminative thoughts. Similarly, dysphoric participants have been shown to have a greater tendency to experience mind wandering (e.g. Smallwood, O’ Connor, Sudbery \& Obonsawin, 2007). Mind wandering, which has been defined as "a shift in the focus of attention away from the here and now towards one's private thoughts and feelings" (Smallwood et al., 2007 page 818), is associated with a decoupling from task-relevant processing. Thus, during the 15 second delay, rather than staying focused on the task in hand (maintaining the faces in working memory) the dysphoric participants in the present study may have become distracted by non-task related processing. Further, there is evidence that depression is associated with impaired motivation (e.g. Schmand et al., 1994), thus the dysphoric group may have been less motivated to maintain focus during the delay period. However, if there was a significant difference in the motivation of the two groups then group differences on the neuropsychological tasks would have been expected, most notably on the digit symbol substitution task. Future work should aim to clarify the contribution of these factors to the observed memory deficits in short-term memory for emotional faces in depressed states. 
A methodological strength of the present study concerns the control of state and trait anxiety, thus the observed group differences cannot be due to comorbid anxiety. Likewise, the inclusion of the battery of neuropsychological tests provides good evidence that the observed changes in face processing were not a consequence of general cognitive impairment in the dysphoric group. However, there are a number of methodological issues that need to be mentioned. Firstly, the sample sizes were relatively small, although previous studies have used smaller samples to demonstrate the required memory effects. Secondly, as participants were classified into discrete categories of dysphoric and non-dysphoric controls based upon a single measure of depressed mood it is unclear whether the depression-associated memory biases are due to state-dependent effects of dysphoria or more stable characteristics of individuals who are vulnerable to depression. Thirdly, the method of allocating participants to groups could have been improved, as a person scoring 9 on the BDI would have been in the non-dysphoric group and someone scoring 10 would have been in the dysphoric group. Future, studies should aim to create more distinct groups. Nevertheless, the procedure utilised in the current study has been used in previous published work (Walker et al., 2003) and was able to produce clear differences in performance on the face processing tasks. Finally, although the experimental groups did not differ significantly in relative proportions of male and female participants, the sample was predominantly female. Recent research has indicated gender differences in mood-congruent memory biases (Gilboa et al.., 2002) therefore studies employing more evenly matched gender groups of dysphoric and control individuals are awaited to validate the current findings.

In summary, dysphoric individuals exhibited significantly impaired memory for facial expression and facial identity relative to non-dysphoric controls. However, 
they also demonstrated some evidence of a mood congruent bias for faces with a negative expression and a mood incongruent memory bias away from faces with happy facial expression. These findings are somewhat consistent with studies that assessed long-term memory for emotional faces in depression, suggesting that the MCM bias observed in long-term memory tasks generalises to short-term memory. However, this was only evident in memory for identity and not emotional expression. These memory deficits, which cannot be ascribed to co-morbid anxiety or general cognitive impairment in the dysphoric group, could have significant implications for dysphoric individuals' social functioning and hence might represent a risk factor for the development of more serious depressive episodes. 


\section{REFERENCES}

Archer, J., Hay, D. C., \& Young, A. W. (1992). Face processing in psychiatric conditions. Br J Clin Psychol, 31 ( Pt 1), 45-61.

Baumeister, R.F., Bratslavsky, E., Finkenauer, C., \& Vohs, K.D. (2001). Bad is stronger than good. Review of General Psychology, 5, 323-370.

Beck, A. T., Brown, G., \& Steer, R. A. (1996). Beck Depression Inventory II manual. San Antonio, TX: The Psychological Corporation

Billings, L.S., Harrison, D.W., \& Alden, J.D. (1993). Age differences among women in the functional asymmetry for bias in facial affect perception. Bulletin of the Psychonomic Society, 31, 317-320.

Bradley, B. P., \& Mogg, K. (1994). Mood and personality in recall of positive and negative information. Behav Res Ther, 32(1), 137-141.

Bradley, B. P., Mogg, K., \& Williams, R. (1995). Implicit and explicit memory for emotion-congruent information in clinical depression and anxiety. Behav Res Ther, 33(7), 755-770.

Bradley, B. P., Mogg, K., Millar, N., Bonham-Carter, C., Fergusson, E., Jenkins, J., \& Parr, M. (1997). Attentional biases for emotional faces. Cognition and Emotion, 11, 25-42.

Brown, R. G., Scott, L. C., Bench, C. J., \& Dolan, R. J. (1994). Cognitive function in depression: its relationship to the presence and severity of intellectual decline. Psychological Medicine, 24(4), 829-847. 
Bruce, V., \& Young, A. W. (1986). Understanding face recognition. Br. J. Psychol, 77, 305-327.

Buchanan, T., \& Adolphs, R. (2002) The role of the human amygdale in emotional modulation of long-term declarative memory. In Moore, S., \& Oaksford, M. (Eds.), Emotional cognition: From brain to behavior (pp. 9-34). London: Benjamins.

Channon, S., \& Green, P. S. (1999). Executive function in depression: the role of performance strategies in aiding depressed and non-depressed participants. $J$ Neurol Neurosurg Psychiatry, 66(2), 162-171.

Cohen-Pager, D., \& Brosgole, L. (1992). The recognition of faces and expressions. Bulletin of the Psychonomic Society, 30, 191-193.

Crews, W. D., \& Harrison, D. W. (1994). Cerebral asymmetry in the facial affect perception of women: Neuropsychological effects of depressed mood. Percept Mot Skills, 79, 1667-1679.

D’Argembeau, A., Van der Linden, M., Comblain, C., \& Etienne, A-M. (2003). The effects of happy and angry expressions on identity and expression memory for unfamiliar faces. Cognition \& Emotion, 17, 609-622.

Deveney, C. M., \& Deldin, P. J. (2004). Memory of faces: a slow wave ERP study of major depression. Emotion, 4(3), 295-304.

Direnfeld, D. M., \& Roberts, J. E. (2006). Mood congruent memory in dysphoria: the roles of state affect and cognitive style. Behav Res Ther, 44(9), 1275-1285.

Eastwood, J. D., Smilek, D., \& Merikle, P. M. (2003). Negative facial expression captures attention and disrupts performance. Percept Psychophys, 65, 352358. 
Ekman, P. \& Friesen, W. V. (1975). Unmasking the face. A guide to recognizing emotions from facial clues. Englewood Cliffs, New Jersey: Prentice-Hall.

Fabre-Thorpe, M., Delorme, A., Marlot, C., \& Thorpe, S. J. (2001). A limit to the speed of processing in Ultra-Rapid Visual Categorization of novel natural scenes. J Cogn Neurosci, 13, 171-180

Foa, E. B., Gilboa-Schechtman, E., Amir, N., \& Freshman, M. (2000). Memory bias in generalized social phobia: Remembering negative emotional expressions. Journal of Anxiety Disorders, 14, 501-519.

Fox, E., Lester, V., Russo, R., Bowles, R. J., Pichler, A., \& Dutton, K. (2000). Facial expressions of emotion: Are angry faces detected more efficiently? Cognition \& Emotion, 14, 61-92.

Frewen, P. A., \& Dozois, D. J. A. (2005). Recognition and interpretation of facial expressions in dysphoric women. Journal of Psychopathology and Behavioral Assessment, 27(4), 305 - 315.

Gilboa, E., \& Gotlib, I. H. (1997). Cognitive biases and affect persistence in previously dysphoric and never-dysphoric individuals. Cognition and Emotion, 11, 517-538.

Gilboa-Schechtman, E., Erhard-Weiss, D., \& Jeczemien, P. (2002). Interpersonal deficits meet cognitive biases: memory for facial expressions in depressed and anxious men and women. Psychiatry Res, 113(3), 279-293.

Gotlib, I. H., Krasnoperova, E., Yue, D. N., \& Joormann, J. (2004a). Attentional biases for negative interpersonal stimuli in clinical depression. J Abnorm Psychol, 113(1), 121-135. 
Gotlib, I. H. Gotlib, I. H., Kasch, K. L., Traill, S., Joormann, J., Arnow, B. A., \& Johnson, S. L. (2004b). Coherence and specificity of information-processing biases in depression and social phobia. J Abnorm Psychol, 113(3), 386-398.

Gotlib, I. H., \& Lee, C. M. (1989). The social functioning of depressed patients: A longitudinal assessment. Journal of Social and Clinical Psychology, 8, 223237.

Hamann, S. (2001). Cognitive and neural mechanisms of emotional memory. Trends Cogn Sci, 5, 394-400.

Harkness, K. L., Sabbagh, M. A., Jacobson, J. A., Chowdrey, N. K., \& Chen, T. (2005) Enhanced accuracy of mental state decoding in dysphoric college students Cognition and Emotion, 19(7), 999-1025

Hartlage, S., Alloy, L. B., Vazquez, C., \& Dykman, B. (1993). Automatic and effortful processing in depression. Psychol Bull, 113(2), 247-278.

Haxby, J. V., Hoffman, E. A., \& Gobbini, . I. (2000).The distributed human neural system for face perception. Trends Cogn Sci, 4, 223-233.

Hipkins, J., Whitworth, M., Tarrier, N., \& Jayson, G. (2004). Social support, anxiety and depression after chemotherapy for ovarian cancer: a prospective study. Br JHealth Psychol, 9(Pt 4), 569-581.

Hugdahl, K., Iversen, P.M., \& Johnsen, B.H. (1993). Laterality for facial expressions: Does the sex of the subjects interact with the sex of the stimulus face? Cortex, 29, 325-331.

Ilsley, J. E., Moffoot, A. P., \& O' Carroll, R. E. (1995). An analysis of memory dysfunction in major depression. J of Affect Disord, 35, 1-9. 
Jermann, F., van der Linden, M., \& D'Argembeau, A. (2008). Identity recognition and happy and sad facial expression recall: influence of depressive symptoms. Memory, 16(4), 364-373.

Jogems-Kosterman, B. J., Zitman, F. G., Van Hoof, J. J., \& Hulstijn, W. (2001). Psychomotor slowing and planning deficits in schizophrenia. Schizophr Res 48, 317-333.

Johnson, S. M., Makinen, J. A., \& Millikin, J. W. (2001). Attachment injuries in couple relationships: A new perspective on impasses in couple therapy. $J$ Marital Fam Ther, 27, 145-155.

Joiner, Jr. T.E., Coyne, J.C. (Eds.), (1999). The Interactional Nature of Depression: Advances in Interpersonal Approaches. American Psychological Association, Washington, DC.

Joormann, J., \& Gotlib, I. H. (2008). Updating the contents of working memory in depression: interference from irrelevant negative material. J Abnorm Psychol, 117(1), 182-192.

Keltner, D., Ekman, P., Gonzaga, G. C., \& Beer, J. (2003). Facial expression of emotion. In R. J. Davidson, K. R. Scherer, \& H. H. Goldsmith (Eds.). Handbook of affective sciences (pp. 415-432). Oxford: Oxford University Press.

Kendall. P., Hollon, S., \& Beck, A. (1987). Issues and recommendations regarding use of the Beck Depression Inventory. Cognitive Ther Res 11, 289299.

Kenrick, D. T., Sadalla, E. K., \& Keefe, R. C. (1998). Evolutionary cognitive psychology: The missing heart of modern cognitive science. In C. Crawford 
\& D.L. Krebs (Eds.), Handbook of Evolutionary Psychology: Ideas, issues, and applications. Mahwah, NJ: Lawrence Erlbaum Associates.

Kensinger, E. A., \& Corkin, S. (2003). Memory enhancement for emotional words: Are emotional words more vividly remembered than neutral words? Cognition \& emotion, 31, 1169-1180

Keppel, G., \& Wickens, T. D. (2004). Design and analysis: A researcher's handbook (4th ed.). New Jersey, USA: Pearson Education, Inc.

Klineberg, E., Clark, C., Bhui, K. S., Haines, M. M., Viner, R. M., Head, J., et al.. (2006). Social support, ethnicity and mental health in adolescents. Soc Psychiatry Psychiatr Epidemiol, 41(9), 755-760.

Kottoor, T. M. (1989). Recognition of faces by adults. Psychological Studies, 34, $102-$ 105

Lee, T. C., Yang, Y. K., Chen, P. S., Hung, N. C., Lin, S. H., Chang, F. L., et al.. (2006). Different dimensions of social support for the caregivers of patients with schizophrenia: main effect and stress-buffering models. Psychiatry Clin Neurosci, 60(5), 546-550.

Leppanen, J. M., Milders, M., Bell, J. S., Terriere, E., \& Hietanen, J. K. (2004). Depression biases the recognition of emotionally neutral faces. Psychiatry Res, 128(2), 123-133.

Mathews, A., \& Bradley, B. (1983). Mood and the self-reference bias in recall. Behav Res Ther, 21(3), 233-239.

Matt, G. E., Vazquez, C., \& Campbell, W. K. (1992). Mood-congruent recall of affectively toned stimuli: A meta-analytic review. Clin Psychol Rev, 12, 227-255. 
McCann, C. D., \& Lalonde, R. N. (1993). Dysfunctional communication and depression: social cognitive processes. American Behavioral Scientist, 36, $271-287$

Nezlek, J. B., Hampton, C. A., \& Shean, G. D. (2000). Clinical depression and everyday social interaction in a community sample. J Abnorm Psychol, 109, 111-19.

Nolen-Hoeksema, S. (1991). Responses to depression and their effects on the duration of depressive episodes. J Abnorm Psychol, 100(4), 569-582.

Nolen-Hoeksema, S., Parker, L. E., \& Larson, J. (1994). Ruminative coping with depressed mood following loss. J Pers Soc Psychol, 67(1), 92-104.

Ohman, A., Lundqvist, D., \& Esteves, F. (2001). The face in the crowd revisited: A threat advantage with schematic stimuli. J Pers Soc Psychol, 80, 381-396.

Palmer, B. W., Boone, K. B., Lesser, I. M., Wohl, M. A., Berman, N., \& Miller, B. L. (1996). Neuropsychological deficits among older patients with MD with Predominantly psychological or vegetative symptoms. Journal of Affective Disorders, 41(1), 17-24

Persad, S. M., \& Polivy, J. (1993). Differences between depressed and nondepressed individuals in the recognition of and response to facial emotional cues. J Abnorm Psychol, 102(3), 358-368.

Petty, S. C., Sachs-Ericsson, N., \& Joiner, T. E. (2004) Interpersonal functioning deficits: temporal or stable characteristics of depressed individuals? Journal of Affective Disorders, 81, 115 - 122

Phillips, M. L. (2004) Facial processing deficits and social dysfunction: how are they 
related? Brain 127(8) 1691-1692

Ridout, N., Astell, A. J., Reid, I. C., Glen, T., \& O'Carroll, R. E. (2003). Memory bias for emotional facial expressions in major depression. Cognition and Emotion, 17(1), 101 - 122.

Ridout, N., Dritschel, B., Matthews, K. McVicar, M., Reid, I. C. \& O' Carroll, R. E. (2009) Memory for emotional faces in major depression following judgement of physical facial characteristics at encoding Cognition and Emotion 23(4) 739-752

Ridout, N., Noreen, A., \& Johal, J. (in press) Memory for emotional faces in naturally occurring dysphoria and induced sadness Behaviour, Research \& Therapy

Sato, W., Kochiyama, T., Yoshikawa, S., \& Matsumura, M. (2001). Emotional expression boosts early visual processing of the face: ERP recording and its decomposition by independent component analysis. Neuro Report. 12, 709714.

Schmand, B., Kuipers, T., Van der Gaag, M., Bosveld, J., Bulthuis, F., \& Jellema, M. (1994). Cognitive disorders and negative symptoms as correlates of motivational deficits in psychotic patients. Psychol Med, 24(4), 869-884.

Segrin, C. (2000). Social skills deficits associated with depression. Clin Psychol Rev, $20,379-403$

Smallwood, J., O’ Connor, R. C., Sudbery, M. V., \& Obonsawin, M. (2007) Mindwandering and dysphoria Cognition and Emotion, 21(4), 816-842

Spielberger, C. D. (1983). Manual for the State-Trait Anxiety Inventory (STAI). PaloAlto, CA: Consulting Psychologists Press 
Surguladze, S. A., Young, A. W., Senior, C., Brebion, G., Travis, M. J., \& Phillips, M. L. (2004). Recognition accuracy and response bias to happy and sad facial expressions in patients with major depression. Neuropsychology, $18(2), 212-218$.

Tarsia, M., Power, M. J., \& Sanavio, E. (2003). Implicit and explicit memory biases in mixed anxiety-depression. J Affect Disord, 77(3), 213-225.

Vaananen, A., Vahtera, J., Pentti, J., \& Kivimaki, M. (2005). Sources of social support as determinants of psychiatric morbidity after severe life events: prospective cohort study of female employees. J Psychosom Res, 58(5), 459467.

Walker, W. R., Skowronski, J. J., Gibbons, J. A., Vogl, R. J., \& Thompson, C. P. (2003). On the emotions that accompany autobiographical memories: Dysphoria disrupts the fading affect bias. Cognition and Emotion, 17 (5), 703- 723.

Watkins, E., \& Brown, R. G. (2002). Rumination and executive function in depression: an experimental study. J Neurol Neurosurg Psychiatry, 72(3), 400402.

Wechsler, D. (1981). Wechsler Adult Intelligence Scale-Revised. Psychological Corporation, New York. 


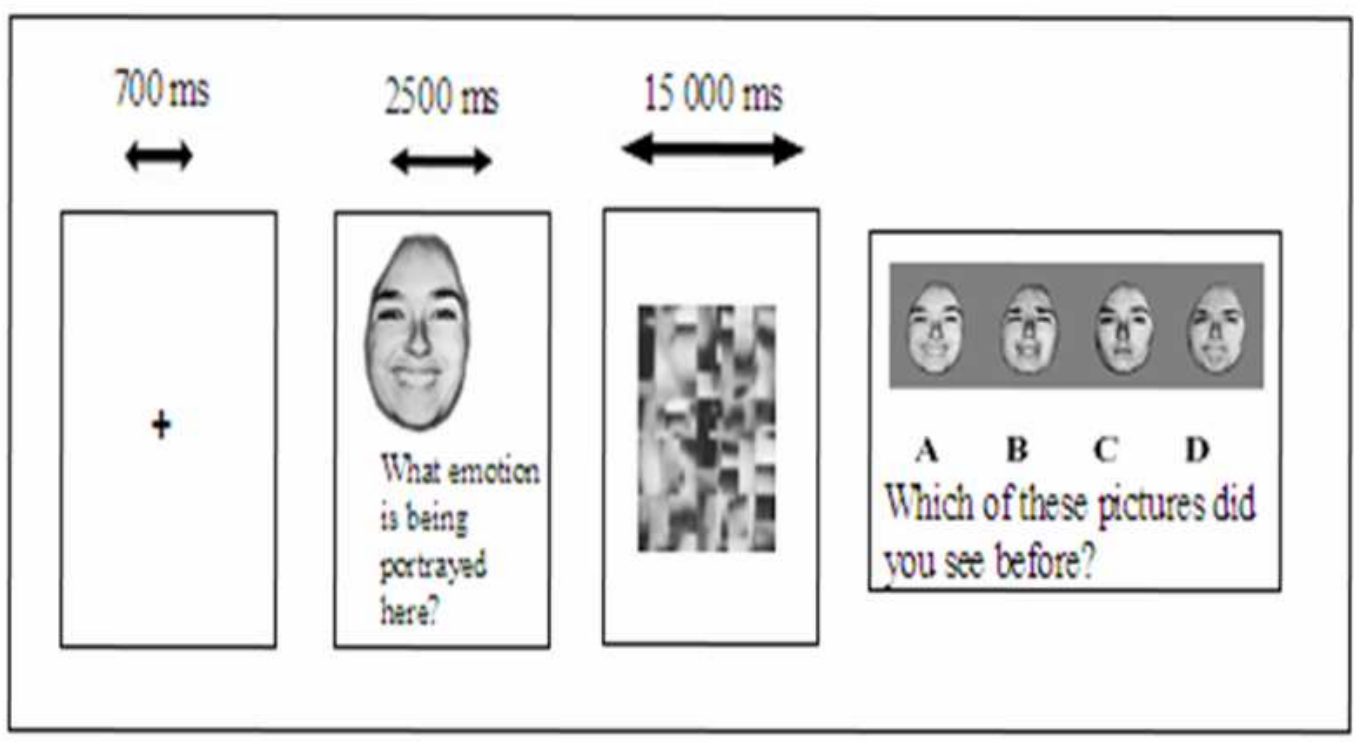

Figure 1. Schematic representation of a single trial of the facial delayedmatching-to-sample task 


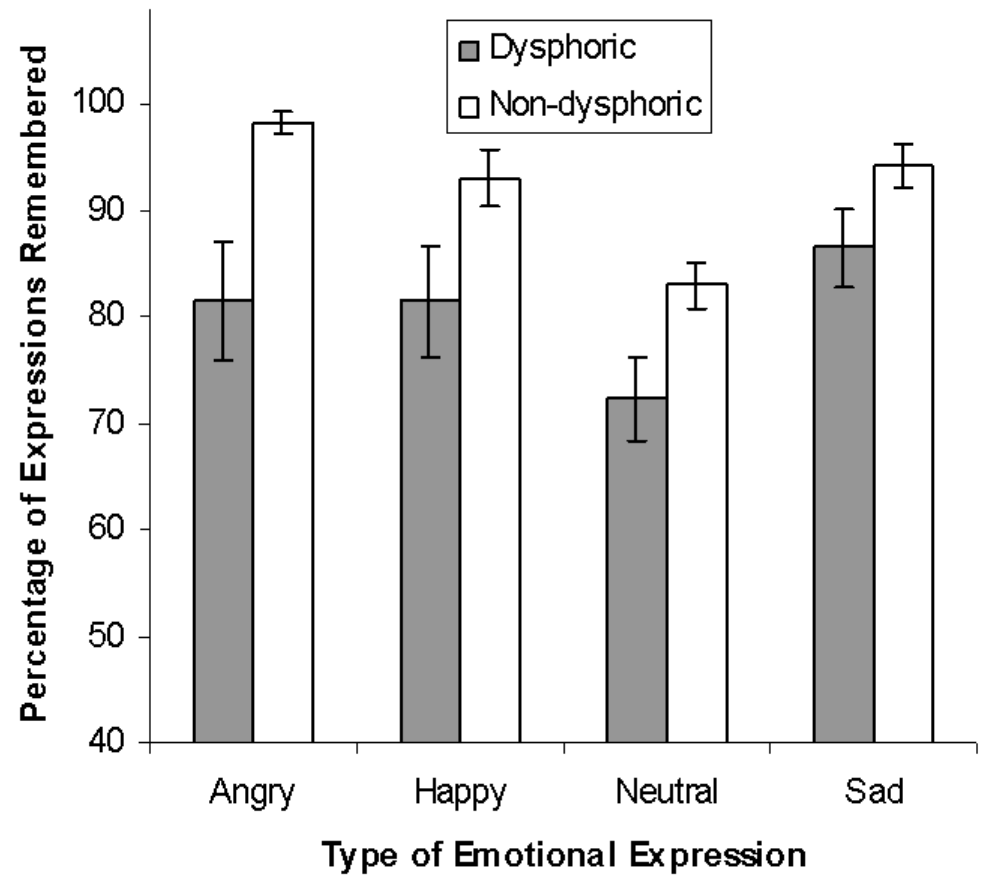

Figure 2. Percentage of emotional expressions correctly recognised by the two groups, as a function of the type of emotion portrayed (error bars show \pm 1 standard error of the mean) 


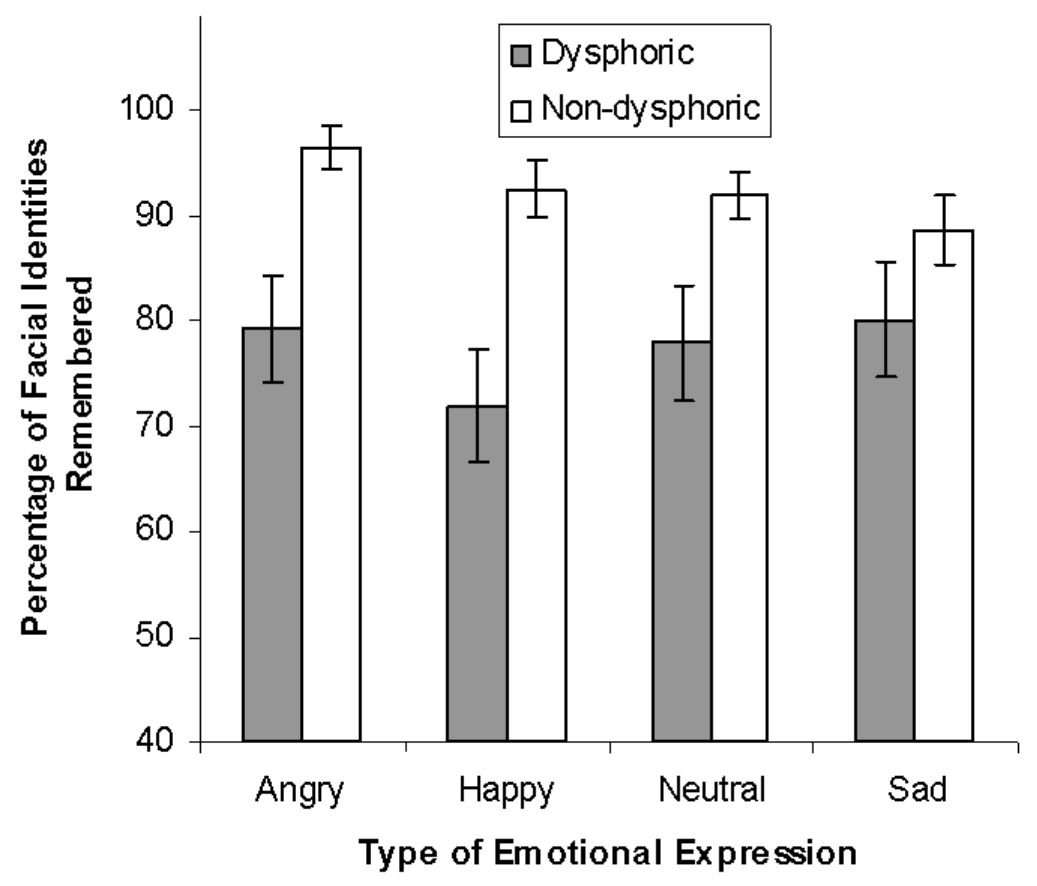

Figure 3. Percentage of facial identities correctly recognised by the two groups, as a function of the type of emotion portrayed (error bars show \pm 1 standard error of the mean) 
Table 1. Participant characteristics (standard deviations are presented in parentheses)

\begin{tabular}{lcc}
\hline & Dysphoric & Non-dysphoric \\
& $(\mathbf{n = 2 9})$ & $(\mathbf{n = 2 2})$ \\
\hline Age & $23.3(2.9)$ & $22.5(3.0)$ \\
Gender ratio (F/M) & $20 / 9$ & $14 / 8$ \\
Depression (BDI)* & $17.8(7.2)$ & $4.9(4.1)$ \\
State Anxiety (STAI-S) & $41.0(11.9)$ & $37.7(9.7)$ \\
Trait Anxiety (STAI-T) & $41.7(7.2)$ & $39.2(9.4)$ \\
\hline$*$ p $<.001$ & &
\end{tabular}


Table 2. Mean performance indices on the neuropsychological test battery, as a function of participant group (standard errors are presented in parentheses)

\begin{tabular}{lcc}
\hline & Dysphoric & Non-dysphoric \\
& $(\mathbf{n}=\mathbf{2 9})$ & $(\mathbf{n = 2 2})$ \\
\hline Digit-span $^{1}$ & $8.9(0.3)$ & $9.2(0.4)$ \\
Digit-span $^{2}$ & $6.6(0.4)$ & $6.1(0.5)$ \\
Spatial-span $^{1}$ & $4.6(0.3)$ & $4.9(0.4)$ \\
Spatial-span $^{2}$ & $5.6(0.2)$ & $5.5(0.3)$ \\
Digit symbol task $^{2}$ & $56.6(2.1)$ & $60.2(2.4)$ \\
Verbal fluency $^{2}$ & $40.9(3.1)$ & $40.6(3.6)$ \\
\hline
\end{tabular}

\footnotetext{
${ }^{1}=$ Forwards Task; ${ }^{2}=$ Backwards Task
} 